\title{
Quantum interference effects in laser spectroscopy of muonic hydrogen, deuterium, and helium-3
}

\author{
Pedro Amaro, ${ }^{1, *}$ Beatrice Franke, ${ }^{2, \dagger}$ Julian J. Krauth, ${ }^{2}$ Marc Diepold, ${ }^{2}$ Filippo Fratini, ${ }^{3}$ Laleh Safari, ${ }^{4}$ Jorge Machado, ${ }^{1,5}$ \\ Aldo Antognini, ${ }^{6,7}$ Franz Kottmann, ${ }^{6}$ Paul Indelicato, ${ }^{5}$ Randolf Pohl, ${ }^{2}$ and José Paulo Santos ${ }^{1}$ \\ ${ }^{1}$ Laboratório de Instrumentação, Engenharia Biomédica e Física da Radiação (LIBPhys-UNL), Departamento de Física, Faculdade de \\ Ciências e Tecnologia, FCT, Universidade Nova de Lisboa, P-2829-516 Caparica, Portugal \\ ${ }^{2}$ Max-Planck-Institute of Quantum Optics, D-85748 Garching, Germany \\ ${ }^{3}$ Atominstitut, Vienna University of Technology, A-1020 Vienna, Austria \\ ${ }^{4}$ Institute of Science and Technology, IST, Austria, Am Campus 1, 3400 Klosterneuburg, Austria \\ ${ }^{5}$ Laboratoire Kastler Brossel, École Normale Supérieure, CNRS, Université P. et M. Curie - Paris 6, Case 74; 4, Place Jussieu, \\ F-75252 Paris CEDEX 05, France \\ ${ }^{6}$ Institute for Particle Physics, ETH Zurich, $\mathrm{CH}-8093$ Zurich, Switzerland \\ ${ }^{7}$ Paul Scherrer Institute, CH-5232 Villigen-PSI, Switzerland
}

(Received 9 June 2015; published 28 August 2015)

\begin{abstract}
Quantum interference between energetically close states is theoretically investigated, with the state structure being observed via laser spectroscopy. In this work, we focus on hyperfine states of selected hydrogenic muonic isotopes, and on how quantum interference affects the measured Lamb shift. The process of photon excitation and subsequent photon decay is implemented within the framework of nonrelativistic second-order perturbation theory. Due to its experimental interest, calculations are performed for muonic hydrogen, deuterium, and helium-3. We restrict our analysis to the case of photon scattering by incident linear polarized photons and the polarization of the scattered photons not being observed. We conclude that while quantum interference effects can be safely neglected in muonic hydrogen and helium-3, in the case of muonic deuterium there are resonances with close proximity, where quantum interference effects can induce shifts up to a few percent of the linewidth, assuming a pointlike detector. However, by taking into account the geometry of the setup used by the CREMA collaboration, this effect is reduced to less than $0.2 \%$ of the linewidth in all possible cases, which makes it irrelevant at the present level of accuracy.
\end{abstract}

DOI: 10.1103/PhysRevA.92.022514

PACS number(s): 32.70.Jz, 36.10.Ee, 32.10.Fn, 32.80.Wr

\section{INTRODUCTION}

Quantum interference (QI) corrections were introduced in the seminal work of Low [1], where the quantum electrodynamics (QED) theory of the natural line profile in atomic physics was formulated. These corrections go beyond the resonant approximation and set a limit for which a standard Lorentzian line profile can be used to describe a resonance. Often referred to as nonresonant corrections [2-4], they contain the full quantum interference between the main resonant channel and other nonresonant channels, which leads to an asymmetry of the line profile. Therefore, the fitting of spectroscopy data with Lorentzian profiles becomes ambiguous, since it leads to energy shifts that depend on the measurement process itself [2-5]. A careful analysis of the limits of the resonance approximation is thus mandatory for high-precision optical and microwave spectroscopy experiments.

The first calculation of QI was made for the Lamb shift in hydrogenlike (H-like) ions and for the photon scattering case [1]. It was found to be relatively small, of the order of $\delta_{\mathrm{QI}} / \Gamma \approx \alpha(\alpha Z)^{2}$, compared to the linewidth $\Gamma$. Here $\delta_{\mathrm{QI}}$ is the line shift due to QI, $\alpha$ is the fine structure constant, and $Z$ is the atomic number. Thus, for some time, little interest has been addressed to these corrections in optical measurements of H-like ions. Since the late 1990s, high-precision measurements of the $1 s-2 s$ transition frequency in hydrogen renewed the

\footnotetext{
*pdamaro@fct.unl.pt

†beatrice.franke@mpq.mpg.de
}

interest in these corrections [6-8]. Numerous theoretical calculations of QI were then made for $\mathrm{H}$-like ions $[3,4,9,10]$ with astrophysical interest [11] and application to laser-dressed atoms [12]. QI has also been studied in other atomic systems and processes during the last decades, mainly because near and crossed resonances of hyperfine states can enhance the QI effects [13-15]. QI effects [16-19] have been shown to be responsible for discrepant measurements of the helium fine structure [20,21] and the lithium charge radii determined by the isotope shift [5]. The lithium experiment [5] gives a beautiful experimental demonstration of the geometry dependence of the QI effect. Very recently, QI effects in two-photon frequencycomb spectroscopy have been investigated, too [22].

In this work, we calculate the QI shifts for $2 s \rightarrow 2 p$ transitions in H-like muonic atoms with hyperfine structure. The physical process considered here is the photon scattering of initial $2 s$ states to final $1 s$ states, with an incident photon energy that is resonant with intermediate $2 p$ states. Figure 1 recalls the $2 s \rightarrow 2 p$ level structures that are considered in this work. The level structures and linewidths [23,25] of the $2 p$ states are given in Table I. The theoretical formalism used here can be traced back to recent works [5,26,27].

Hyperfine states in $\mu^{1} \mathrm{H}$ are separated by several hundred $\mathrm{GHz}$ [23,28-30], and have linewidths of a few tens of $\mathrm{GHz}$ [25]. Thus, it is expected that QI plays a small role and cannot be responsible for the so-called "proton radius puzzle," where a discrepancy of four linewidths was observed in the experiments of the Charge Radius Experiment with Muonic Atoms (CREMA) collaboration [31,32]. Nevertheless, these systematics need to be carefully evaluated and quantified, since 

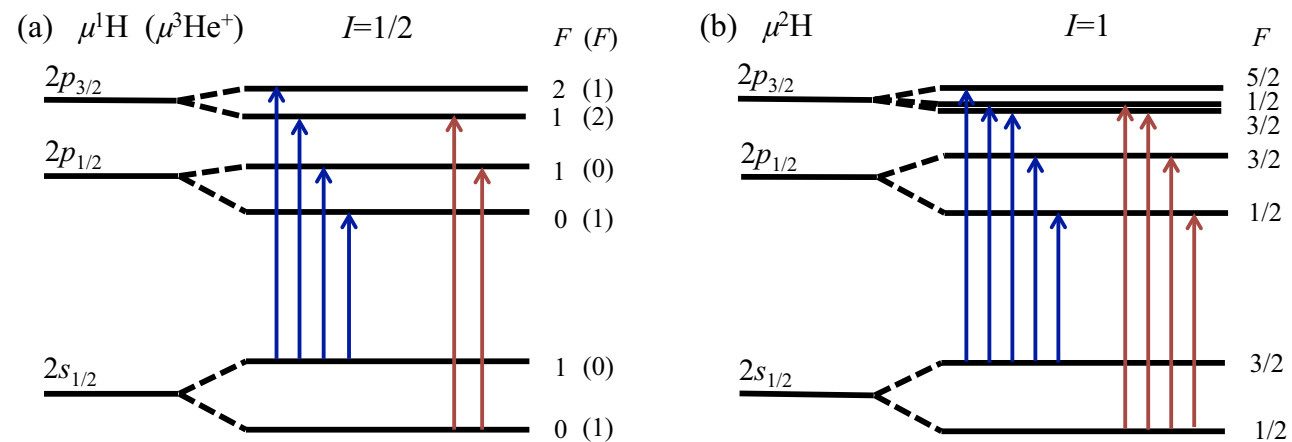

FIG. 1. (Color online) $2 s$ and $2 p$ level structure (not in scale) for $\mu^{1} \mathrm{H}, \mu^{3} \mathrm{He}^{+}$(a), and $\mu^{2} \mathrm{H}$ (b). Allowed electric dipole transitions are also shown. $I$ and $F$ are the nuclear spin and total angular momentum, respectively.

they have contributions similar to small QED corrections (e.g., sixth-order contributions [23]) and thus may impact precise determinations of the proton charge radius. Since resonances in $\mu^{3} \mathrm{He}^{+}[33,34]$ have been measured recently, values of the corresponding QI contributions are presented here, too. In the case of $\mu^{2} \mathrm{H}$, there is a close energy proximity between the states $2 p_{3 / 2}^{F=1 / 2}$ and $2 p_{3 / 2}^{F=3 / 2}$ of $\approx 87 \mathrm{GHz}$ and hence, it is expected that QI effects could be much higher.

\section{THEORY}

Photon scattering is a two-step process consisting of photon excitation with subsequent photon decay, which is formally equivalent to Raman anti-Stokes scattering. It is described by second-order theories (e.g., Kramers-Heisenberg formula [35], or $S$ matrix [36]), which overall converge to the following

TABLE I. Calculated energy differences of $\mu^{1} \mathrm{H}, \mu^{2} \mathrm{H}$, and $\mu^{3} \mathrm{He}^{+}[23,24]$ states relative to the lowest $n=2$ hyperfine state, which are $2 s_{1 / 2}^{F=0}, 2 s_{1 / 2}^{F=1 / 2}$, and $2 s_{1 / 2}^{F=1}$, respectively. $\Gamma_{2 p}$ is the respective approximate linewidth.

\begin{tabular}{lccc}
\hline \hline & & $\mathrm{GHz}$ & $\mathrm{meV}$ \\
\hline$\mu^{1} \mathrm{H}$ & $2 s_{1 / 2}(F=1)$ & 5520 & 22.83 \\
& $2 p_{1 / 2}(F=0)$ & 51631 & 213.53 \\
& $2 p_{1 / 2}(F=1)$ & 53521 & 221.35 \\
& $2 p_{3 / 2}(F=1)$ & 54616 & 225.88 \\
& $2 p_{3 / 2}(F=2)$ & 55402 & 229.12 \\
& $\Gamma_{2 p}[25]$ & 18.5 & 0.0765 \\
$\mu^{2} \mathrm{H}$ & $2 s_{1 / 2}(F=3 / 2)$ & 1485 & 6.143 \\
& $2 p_{1 / 2}(F=1 / 2)$ & 49680 & 205.46 \\
& $2 p_{1 / 2}(F=3 / 2)$ & 50182 & 207.54 \\
& $2 p_{3 / 2}(F=3 / 2)$ & 52016 & 215.12 \\
& $2 p_{3 / 2}(F=1 / 2)$ & 52104 & 215.49 \\
& $2 p_{3 / 2}(F=5 / 2)$ & 52286 & 216.24 \\
$\mu^{3} \mathrm{He}$ & $2 s_{1 / 2}(25]$ & 19.5 & 0.0806 \\
& $2 p_{1 / 2}(F=1)$ & 41443 & 171.40 \\
& $2 p_{1 / 2}(F=0)$ & 325633 & 1288.08 \\
& $2 p_{3 / 2}(F=2)$ & 347860 & 1438.63 \\
& $2 p_{3 / 2}(F=1)$ & 353731 & 1462.92 \\
& $\Gamma_{2 p}{ }^{\mathrm{a}}$ & 318.7 & 0.1318 \\
\hline \hline
\end{tabular}

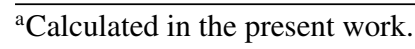

scattering amplitude (velocity gauge and atomic units) from initial to final states $[26,35,36]$,

$$
\begin{aligned}
\mathcal{M}_{i \rightarrow f}^{\varepsilon_{1} \boldsymbol{\varepsilon}_{2}}= & \sum_{\nu}\left[\frac{\left\langle f\left|\alpha \boldsymbol{p} \cdot \boldsymbol{\varepsilon}_{2}\right| \nu\right\rangle\left\langle\nu\left|\alpha \boldsymbol{p} \cdot \boldsymbol{\varepsilon}_{1}\right| i\right\rangle}{\omega_{\nu i}-\omega_{1}-i \Gamma_{\nu} / 2}\right. \\
& \left.+\frac{\left\langle f\left|\alpha \boldsymbol{p} \cdot \boldsymbol{\varepsilon}_{1}\right| \nu\right\rangle\left\langle\nu\left|\alpha \boldsymbol{p} \cdot \boldsymbol{\varepsilon}_{2}\right| i\right\rangle}{\omega_{\nu i}+\omega_{2}-i \Gamma_{\nu} / 2}\right]
\end{aligned}
$$

where $|i\rangle,|v\rangle$, and $|f\rangle$ represent the initial, intermediate, and final hyperfine states of the muonic atom or ion. $\omega_{\nu i}=$ $E_{v}-E_{i}$ is the transition frequency between $|v\rangle$ and $|i\rangle$. The dipole approximation, $\alpha \boldsymbol{p} \cdot \boldsymbol{\varepsilon}_{\gamma}(\gamma=1,2)$ is used, where $\boldsymbol{p}$ is the linear momentum operator and $\boldsymbol{\varepsilon}_{\gamma}$ is the incident (scattered) photon polarization. The summation over the intermediate states $|\nu\rangle$ runs over all solutions of the Dirac spectrum of the muonic atom (ion) with hyperfine structure. All states are considered with a well-defined total atomic angular momentum $F$, projection along the quantified axis $m$, and total orbiting particle angular momentum $J$ [37]; thus the contribution of off-diagonal terms (mixing between the $2 p_{1 / 2}^{F=1}$ and $2 p_{3 / 2}^{F=1}$ states) [38] is considered null. $\Gamma_{\nu}$ is the full

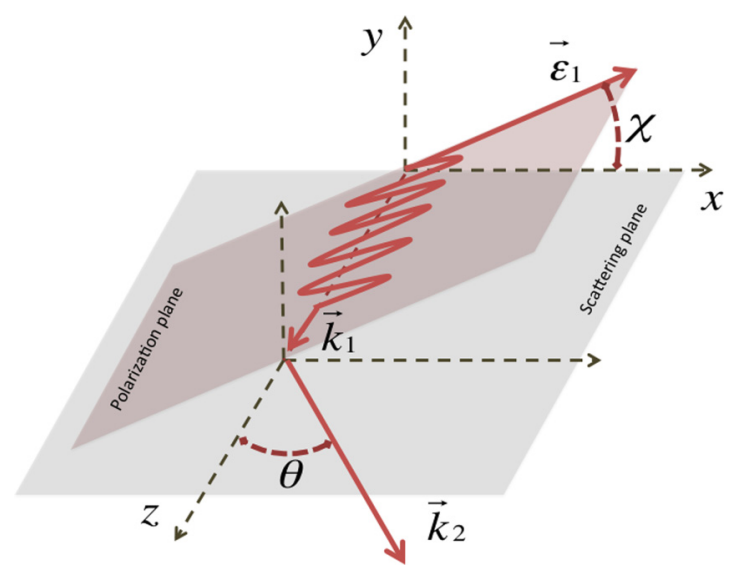

FIG. 2. (Color online) Adopted geometry for photon scattering of incident linear polarized photons with momentum $\boldsymbol{k}_{1}$ and polarization $\boldsymbol{\varepsilon}_{1}$ and scattered photon momentum $\boldsymbol{k}_{2}$, uniquely defined by $\theta$. The scattered photon's polarization is not observed in our measurements and is thus not illustrated. 
width at half maximum for an isolated Lorentzian line of the excited state, where we assume $\Gamma_{2 p_{j}^{F}} \equiv \Gamma_{2 p}$.

The incident photon energies studied in this work comprise the near-resonant region of the $2 s \rightarrow 2 p$ transitions. This includes only the resonances illustrated in Fig. 1. Hence, we restrict the summation $v$ to the $2 p$ states of the terms $\omega_{2 s 2 p}-\omega_{1}$ [first part of the right side of Eq. (1)].

Energy conservation leads to $E_{i}-E_{f}=\omega_{2}-\omega_{1}$ [26] between the initial $\left(E_{i}\right)$ and final $\left(E_{f}\right)$ energy states and the energy of the incident $\left(\omega_{1}\right)$ and scattered $\left(\omega_{2}\right)$ photons; thus only one of the photon energies is independent. Using this relation, it is convenient to introduce the energy sharing parameter $u=\omega_{1} / \omega_{r}$ defined by the fraction of the incident photon energy relative to the lowest resonant energy $\omega_{r}$ of a given muonic atom with initial $F_{i}$ (see Fig. 1).

Motived by the experimental configuration, we consider incident photons having linear polarization and nonobservation of scattered photon's polarization, as illustrated in Fig. 2 [39]. If we define the scattering plane, containing both photon momenta $\left(\boldsymbol{k}_{1}\right.$ and $\left.\boldsymbol{k}_{2}\right)$, then a single polar angle $\theta$ is sufficient for describing the angular distribution of $\boldsymbol{k}_{2}$.

The corresponding differential cross section of the amplitude in Eq. (1) for all the mentioned approximations is given by [36]

$$
\begin{aligned}
& \frac{d \sigma}{d \Omega}(u, \theta, \chi) \\
& \quad=\frac{1}{\left(2 F_{i}+1\right)} \sum_{\substack{m_{i}, F_{f}, m_{f}, J_{f} \\
\boldsymbol{\varepsilon}_{2}}}\left|\mathcal{M}_{i \rightarrow f}^{\varepsilon_{1}, \boldsymbol{\varepsilon}_{2}}\right|^{2} \\
& \quad \approx \frac{\omega_{r}^{2} u u_{f i}^{3}}{\left(2 F_{i}+1\right)} \sum_{\substack{m_{i}, F_{f}, m_{f}, J_{f} \\
\boldsymbol{\varepsilon}_{2}}}\left|\sum_{F_{v}, m_{v}, J_{v}} \frac{D_{F_{i} m_{v} J_{i}}^{F_{v} m_{v} J_{v}}\left(D_{F_{v} m_{v} J_{v}}^{F_{f} m_{f} J_{f}}\right)^{*}}{\bar{\omega}_{v i}-u-i \bar{\Gamma}_{\nu} / 2}\right|^{2},
\end{aligned}
$$

where $\bar{\omega}_{v i}=\omega_{\nu i} / \omega_{r}, \bar{\Gamma}_{v}=\Gamma_{\nu} / \omega_{r}$ and $u_{f i}=u+\bar{\omega}_{f i}$. In Eq. (2), it is assumed that the initial state of the atom is unpolarized and that the level and magnetic sublevels of the final state, as well as the scattered photon's polarization $\left(\boldsymbol{\varepsilon}_{2}\right)$ remains unobserved in the scattering process. $D_{F^{\prime} m^{\prime} J^{\prime}}^{F m J^{\prime}}$ are the dipole matrix elements (length gauge). Equation (2) can be further rearranged as a sum of Lorentzian components $\Lambda_{J_{i} J_{v}}^{F_{i} F_{v}}(\theta, \chi)$, and cross terms $\Xi_{J_{i} J_{v} J_{v}^{\prime}}^{F_{i} F_{v} F_{v}^{\prime}}(\theta, \chi)$, similar to Ref. [5]. For our particular geometry and atomic system, the result is given by

$$
\frac{d \sigma}{d \Omega}(u, \theta, \chi)=\frac{\omega_{r}^{2} u u_{f i}^{3} \mathcal{S}_{f v i}^{2}}{\left(2 F_{i}+1\right)}\left(\sum_{F_{v}, J_{v}} \frac{\Lambda_{J_{i} J_{v}}^{F_{i} F_{v}}(\theta, \chi)}{\left(\bar{\omega}_{v i}-u\right)^{2}+\left(\bar{\Gamma}_{v} / 2\right)^{2}}+\sum_{\left(F_{v}^{\prime}, J_{v}^{\prime}\right)>\left(F_{v}, J_{v}\right)} \frac{\Xi_{J_{i} J_{v} J_{v}^{\prime}}^{F_{i} F_{v} F_{v}^{\prime}}(\theta, \chi)}{\left(\bar{\omega}_{v i}-u-i \bar{\Gamma}_{v} / 2\right)\left(\bar{\omega}_{\nu^{\prime} i}-u+i \bar{\Gamma}_{v} / 2\right)}\right),
$$

where the second summation over $F_{v}^{\prime}$ and $J_{v}^{\prime}$ runs for nonrepeated values of $F_{v}$ and $J_{v}$ of the first summation. The quantities defined by

$$
\Lambda_{J_{i} J_{v}}^{F_{i} F_{v}}(\theta, \chi)=\sum_{m_{i}, F_{f}, m_{f}, J_{f}, \boldsymbol{\varepsilon}_{2}}\left|\Omega_{J_{i} J_{v} J_{f}}^{F_{i} F_{v} F_{f}}\left(\theta, \chi, \boldsymbol{\varepsilon}_{2}\right)\right|^{2},
$$

and

$$
\boldsymbol{\Xi}_{J_{i} J_{v} J_{v}^{\prime}}^{F_{v} F_{v} F_{v}^{\prime}}(\theta, \chi)=2 \operatorname{Re}\left[\sum_{\substack{m_{i}, F_{f} \\ m_{f}, J_{f}, \boldsymbol{\varepsilon}_{2}}} \Omega_{J_{i} J_{v} J_{f}}^{F_{i} F_{v} F_{f}}\left(\theta, \chi, \boldsymbol{\varepsilon}_{2}\right)\left(\Omega_{J_{i} J_{v}^{\prime} J_{f}}^{F_{i} F_{f}^{\prime} F_{f}}\left(\theta, \chi, \boldsymbol{\varepsilon}_{2}\right)\right)^{*}\right],
$$

contain all the polarization and geometrical dependencies. $D_{F^{\prime} m^{\prime} J^{\prime}}^{F m J}, \Omega_{J_{i} J_{v} J_{f}}^{F_{i} F_{v} F_{f}}\left(\theta, \chi, \varepsilon_{2}\right)$ and $\mathcal{S}_{f v i}$ are given in the Appendix.

The differential cross section of Eqs. (2) and (3) contains a coherent summation over resonant excitation channels; thus it takes into account channel interference between neighboring resonances. However, as can be observed in Eq. (3), if cross terms $\Xi$ were removed, it reduces to an incoherent sum of independent Lorentzian profiles. The QI effects are thus included in those cross terms.

\section{RESULTS AND DISCUSSION}

In this section, we present and discuss results for the QI contribution in several muonic atoms taking into account Eqs. (3) and (4), first assuming a pointlike detector and later the CREMA geometry. The influence of the geometric and polarization conditions on the QI is well described in Ref. [5] and is reproduced in the present work. We thus restrict our geometrical settings to the perpendicular observation $\left(\theta=90^{\circ}\right)$ of scattered photons and to the case of horizontally and vertically polarized photons with respect to the scattering plane $\left(\chi=0^{\circ}\right.$ and $\left.\chi=90^{\circ}\right)$. Figure 3 displays the scattering cross section for the $2 s \rightarrow 2 p \rightarrow 1 s$ processes in $\mu^{1} \mathrm{H}$ and $\mu^{2} \mathrm{H}$, on one hand, having the full coherent summation of Eq. (2) (i.e., with QI), on the other hand, having the summation restricted to only the Lorentzian terms [neglecting cross terms in Eq. (3)]. The peaks correspond to the respective transitions shown in Fig. 1. As it is observed, the influence of QI is more noticeable in regions between resonances, where no dominant excitation channels exist. Close to resonances, the influence of QI is approximately equivalent to shifting the peak position, as shown in the zoom plot of Fig. 3 .

We determine this shift in each resonance by generating a pseudospectrum that follows the theoretical profile of Eq. (3) and fitting it with an incoherently sum of Lorentzians (as performed in the data analysis of the CREMA experiments). 
(a)

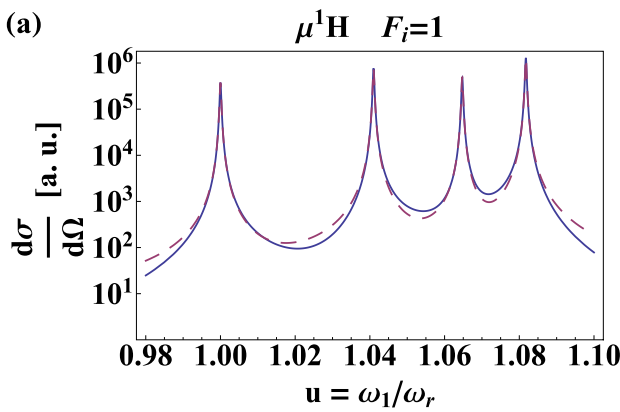

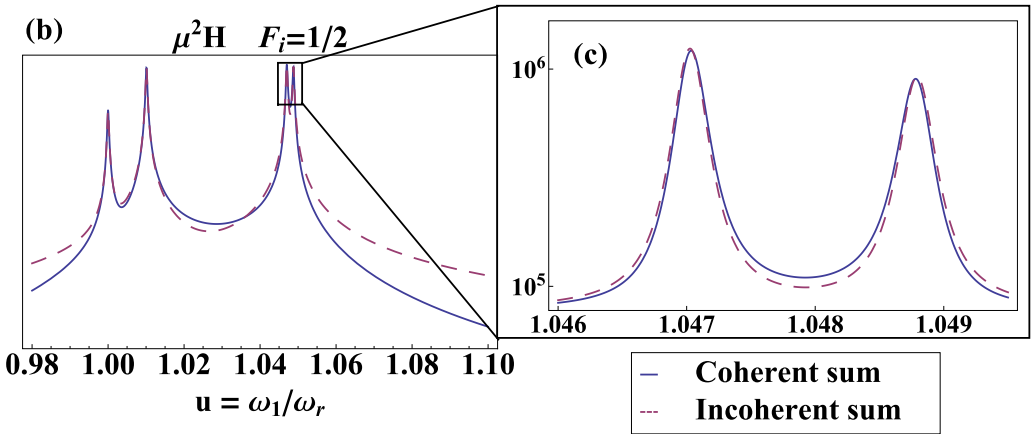

FIG. 3. (Color online) Scattering differential cross section (3) at $\theta=90^{\circ}$ and $\chi=0^{\circ}$ for $\mu^{1} \mathrm{H}$ (a) and $\mu^{2} \mathrm{H}$ (b) versus photon frequency. A zoom plot of the two close-lying resonances for $\mu^{2} \mathrm{H}$ is given in the right panel (c). Solid blue lines correspond to the full evaluation of Eq. (3), while the dashed red curves account for only the first term in the right side of Eq. (3) (sum of Lorentzian profiles).

Fits are done using the ROOT/MINUIT package [40]. All fit parameters (position, amplitude, and linewidth) are free fit parameters for each transition. The fit range is chosen sufficiently large, such that the fit results do not depend on it. The shifts of the fitted resonance position $\delta_{\mathrm{QI}}$, normalized to $\Gamma_{2 p}$, for each resonance and muonic atoms are given in Table II. Overall, with the exception of some resonances in

TABLE II. Shift of the line center due to QI contribution for $\mu^{1} \mathrm{H}, \mu^{3} \mathrm{He}^{+}$, and $\mu^{2} \mathrm{H} .|i\rangle$ and $|\nu\rangle$ stand for the initial and resonant atomic state, respectively. Values of $\delta$ divided by $\Gamma_{2 p}$ are given for a pointlike detector with vertical $\left(\delta_{\mathrm{QI}}^{\perp}, \chi=90^{\circ}, \theta=90^{\circ}\right)$ and horizontal $\left(\delta_{\mathrm{QI}}^{\|}, \chi=0^{\circ}, \theta=90^{\circ}\right)$ polarization cases, as well as for the CREMA setup geometry $\left(\delta_{\mathrm{QI}}^{*}\right)$.

\begin{tabular}{|c|c|c|c|c|c|}
\hline & $|i\rangle$ & $|v\rangle$ & $\delta_{\mathrm{QI}}^{\perp}(\%)$ & $\delta_{\mathrm{QI}}^{\|}(\%)$ & $\delta_{\mathrm{QI}}^{*}(\%)$ \\
\hline \multirow[t]{6}{*}{$\mu^{1} \mathrm{H}$} & \multirow[t]{2}{*}{$2 s_{1 / 2}^{F=0}$} & $2 p_{1 / 2}^{F=1}$ & -0.8 & 1.6 & -0.02 \\
\hline & & $2 p_{3 / 2}^{F=1}$ & 0.4 & -1.7 & 0.01 \\
\hline & \multirow[t]{4}{*}{$2 s_{1 / 2}^{F=1}$} & $2 p_{1 / 2}^{F=0}$ & -0.2 & 0.5 & -0.01 \\
\hline & & $2 p_{1 / 2}^{F=1}$ & -0.6 & 1.2 & -0.01 \\
\hline & & $2 p_{3 / 2}^{F=1}$ & -0.5 & 0.7 & -0.01 \\
\hline & & $2 p_{3 / 2}^{F=2}$ & 0.3 & -1.2 & 0.01 \\
\hline \multirow[t]{6}{*}{$\mu^{3} \mathrm{He}^{+}$} & \multirow[t]{2}{*}{$2 s_{1 / 2}^{F=0}$} & $2 p_{1 / 2}^{F=1}$ & -0.4 & 0.7 & 0.00 \\
\hline & & $2 p_{3 / 2}^{F=1}$ & 0.2 & -0.6 & 0.05 \\
\hline & \multirow[t]{4}{*}{$2 s_{1 / 2}^{F=1}$} & $2 p_{1 / 2}^{F=1}$ & -0.3 & 0.5 & -0.01 \\
\hline & & $2 p_{1 / 2}^{F=0}$ & -0.6 & 1.4 & -0.01 \\
\hline & & $2 p_{3 / 2}^{F=2}$ & -0.1 & 0.4 & 0.00 \\
\hline & & $2 p_{3 / 2}^{F=1}$ & 2.2 & -3.2 & -0.02 \\
\hline \multirow[t]{9}{*}{$\mu^{2} \mathrm{H}$} & \multirow[t]{4}{*}{$2 s_{1 / 2}^{F=1 / 2}$} & $2 p_{1 / 2}^{F=1 / 2}$ & -0.3 & 0.7 & -0.01 \\
\hline & & $2 p_{1 / 2}^{F=3 / 2}$ & -0.5 & 1.0 & -0.01 \\
\hline & & $2 p_{3 / 2}^{F=3 / 2}$ & -3.2 & 6.7. & -0.08 \\
\hline & & $2 p_{3 / 2}^{F=1 / 2}$ & 4.4 & -8.0 & 0.11 \\
\hline & \multirow[t]{5}{*}{$2 s_{1 / 2}^{F=3 / 2}$} & $2 p_{1 / 2}^{F=1 / 2}$ & -0.3 & 0.7 & -0.01 \\
\hline & & $2 p_{1 / 2}^{F=3 / 2}$ & -0.4 & 0.9 & -0.01 \\
\hline & & $2 p_{3 / 2}^{F=3 / 2}$ & -1.2 & 1.9 & -0.03 \\
\hline & & $2 p_{3 / 2}^{F=1 / 2}$ & -4.9 & 12.3 & -0.13 \\
\hline & & $2 p_{3 / 2}^{F=5 / 2}$ & 0.9 & -2.7 & 0.03 \\
\hline
\end{tabular}

$\mu^{2} \mathrm{H}$, QI produces relative shifts $\delta_{\mathrm{QI}}$ less than $3 \%$ of $\Gamma_{2 p}$. For $\mu^{1} \mathrm{H}$ and $\mu^{3} \mathrm{He}^{+}$, the shifts are of the order of $\sim 0.2 \%-1.7 \%$ $(\sim 36-310 \mathrm{MHz})$ and $\sim 0.2 \%-3.2 \%(\sim 0.6-10 \mathrm{GHz})$ of their linewidths, respectively.

The observed discrepancy (proton radius puzzle) of $\sim 75 \mathrm{GHz}(0.31 \mathrm{meV})[31,32]$ at the $2 p_{3 / 2}^{F=2}$ resonance corresponds to four linewidths. Hence, it is much larger than any possible QI contribution. Moreover, this resonance is $\sim 7$ times more intense than the closest resonance $2 p_{3 / 2}^{F=1}$ (see Fig. 3), which minimizes the QI shift in this resonance.

The values presented in Table II for $\mu^{1} \mathrm{H}$ and $\mu^{3} \mathrm{He}^{+}$have the same order as the respective ones given by the rule of thumb for distant resonances $\left(\delta_{\mathrm{QI}} \sim \Gamma_{2 p}{ }^{2} / 4 \Delta\right.$ with $\Delta$ being the energy difference between two resonances) [16]. Apart from this, relatively low intensity resonances, like $2 p_{3 / 2}^{F=1}$ in $\mu^{3} \mathrm{He}^{+}$, can have higher QI contributions due to a high intensity resonance nearby.

On the other hand, the resonances $2 p_{3 / 2}^{F=1 / 2}$ and $2 p_{3 / 2}^{F=3 / 2}$ in $\mu^{2} \mathrm{H}$ are more sensitive to QI effects not only due to their close proximity $(87 \mathrm{GHz}$ ), but also due to the intensities being comparable within a factor of $\sim 0.7$. In this case, the QI shifts can be up to $12 \%$ of $\Gamma_{2 p}(\sim 2 \mathrm{GHz})$.

Applying the previously calculated cross sections to the geometry of the CREMA setup leads to considerable cancellations of the quantum interference effect.

Figure 4 sketches the experimental geometry. Muonic atoms (ions) are formed in an elongated gas volume of $5 \times 12 \times 190 \mathrm{~mm}^{3}$ that is illuminated from the side using a pulsed laser [41,42] and a multipass cavity [43]. The $2 p \rightarrow 1 s$ photons emitted after laser-induced $2 s \rightarrow 2 p$ transitions are detected by two $\mathrm{x}$-ray detectors $\left(14 \times 150 \mathrm{~mm}^{2}\right.$ active area each) [44-46] placed $8 \mathrm{~mm}$ above and below the muon beam axis.

The simplest situation is that the $2 s \rightarrow 2 p$ excitation happens in the center of the target (red circle in Fig. 4), and the resulting $\boldsymbol{k}_{\mathbf{2}}$ photon is detected in the center of the top detector (black circle). This corresponds to the pointlike detector case of $\theta=90^{\circ}$ and $\chi=90^{\circ}\left(\delta_{\mathrm{QI}}^{\perp}\right.$ in Table II).

However, the $2 s \rightarrow 2 p$ excitation takes place anywhere in the muon stop volume, and the photons of the $2 p \rightarrow 1 s$ decay are detected anywhere on the detectors surfaces. We consider here the laser's propagation $\left(\boldsymbol{k}_{1}\right)$ and polarization $\left(\boldsymbol{\varepsilon}_{1}\right)$ directions being along $\boldsymbol{e}_{1}$ and $\boldsymbol{e}_{3}$, respectively. Integrating 


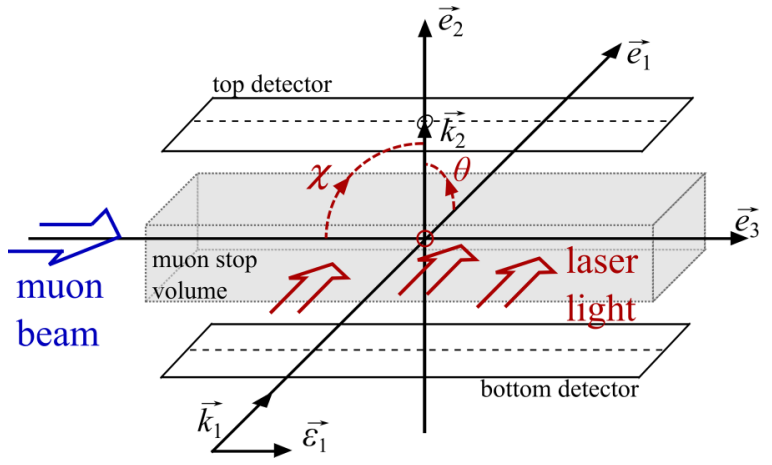

FIG. 4. (Color online) Simplified view of the CREMA target volume. Muons enter the gas target from the left (blue arrow). The gray cuboid indicates the muon stop volume which is illuminated by a laser pulse propagating inside a multipass cavity (red arrows). The incoming direction and polarization are indicated by $\boldsymbol{k}_{\mathbf{1}}$ and $\boldsymbol{\varepsilon}_{\mathbf{1}}$. The pointlike detector assumption treats the case where the muonic atom is at the center (red circle) and the scattered photon $\boldsymbol{k}_{\mathbf{2}}$ is emitted along $\boldsymbol{e}_{2}$ and hits the top detector in the center (black circle).

Eq. (3) over all possible angles $\chi$ accepted by the detector, while averaging over the muon stopping volume results in a considerable reduction of the observed QI effect. Again, we create pseudodata for the real geometry, fit the resonances with a simple sum of Lorentzians, and determine the resulting shift $\delta_{\mathrm{QI}}^{*}$ of the line centers. We notice that taking into account the scattered photons at $\theta \neq 90^{\circ}$ and also the inhomogeneous muon stop probability, hardly affects the final results. As can be seen in Table II, these shifts are much lower than the experimental accuracy of a few percent of the linewidth for all muonic atoms considered here [31,32].

\section{CONCLUSION}

We quantified the line shift caused by quantum interference for $\mu^{1} \mathrm{H}, \mu^{2} \mathrm{H}$, and $\mu^{3} \mathrm{He}^{+}$resonances, assuming first a pointlike detector. For $\mu^{1} \mathrm{H}$, the resulting shifts are small. Hence, quantum interference cannot be the source of the proton radius puzzle, which requires a shift of the resonance in $\mu^{1} \mathrm{H}$ by four linewidths $[31,32]$. On the other hand, the influence of quantum interference for some resonances of $\mu^{2} \mathrm{H}$ can be as large as $12 \%$ of the linewidth for a pointlike detector.

However, we verified that even for those large QI shifts, obtained assuming a pointlike detector, the angular averaging caused by the large acceptance angle of the photon detector and the size of the muon stop volume in the CREMA experiment significantly reduces this effect to negligible values at the present level of accuracy.

\section{ACKNOWLEDGMENTS}

This research was supported in part by Fundação para a Ciência e a Tecnologia (FCT), Portugal, through Projects No. PEstOE/FIS/UI0303/2011 and No. PTDC/FIS/117606/2010, financed by the European Community Fund FEDER through the COMPETE. P.A. and J.M. acknowledge support from the FCT (Portugal), under Contracts No. SFRH/BPD/92329/2013 and No. SFRH/BD/52332/2013. B.F., J.J.K., M.D., and R.P. acknowledge support from the European Research Council
(ERC) (Belgium) through StG. Grant No. 279765. F.F. acknowledges support from the Austrian Science Fund (FWF) (Austria) through START Grant No. Y 591-N16. L.S. acknowledges financial support from the People Programme (Marie Curie Actions) of the European Union's Seventh Framework Programme (FP7/2007-2013) (Belgium) under REA Grant Agreement No. 291734. A.A. acknowledges support from Swiss National Fond (SNF) (Switzerland) Grant No. 200020 159755. B.F. and R.P. would like to thank A. Beyer, L. Meisenbacher, and Th. Udem for illuminating discussions.

\section{APPENDIX: DIPOLE MATRIX ELEMENTS}

The dipole matrix elements $D_{F^{\prime} m^{\prime} J^{\prime}}^{F m J}$ in Eq. (2) are evaluated using standard angular reduction methods, which can start by simply expanding the product of the photon polarization and the position vector $\left(\varepsilon_{\gamma} \cdot \boldsymbol{r}\right)$ in a spherical basis, i.e.,

$$
\begin{aligned}
D_{F^{\prime} m^{\prime} J^{\prime}}^{F m J} & =\left\langle\beta^{\prime} F^{\prime} m^{\prime} J^{\prime}\left|\varepsilon_{\gamma} \cdot \boldsymbol{r}\right| \beta F m J\right\rangle \\
& =\sum_{\lambda=-1}^{1}(-1)^{\lambda} \varepsilon_{\gamma}^{-\lambda}\left\langle\beta^{\prime} F^{\prime} m^{\prime} J^{\prime}\left|r_{\lambda}\right| \beta F m J\right\rangle,
\end{aligned}
$$

where $\beta$ contains all additional quantum numbers of the atomic state besides $F, m$, and $J$. Following the geometry and nomenclature of Fig. 2, the spherical form of the (normalized) polarization vectors are given by

$$
\begin{aligned}
& \varepsilon_{l}^{( \pm 1)}=\mp \frac{\left(\cos \chi_{1} \pm i \sin \chi_{1}\right)}{\sqrt{2}}, \quad \varepsilon_{l}^{(0)}=0, \\
& \varepsilon_{2}^{( \pm 1)}=\mp \frac{\left(\cos \chi_{2} \cos \theta \pm i \sin \chi_{2}\right)}{\sqrt{2}}, \quad \varepsilon_{2}^{(0)}=-\cos \chi_{2} \sin \theta,
\end{aligned}
$$

where $\chi \equiv \chi_{1}$.

The matrix elements of $r_{\lambda}$ can be further simplified by making use of the Wigner-Eckart theorem [47] and considering the overall atomic state being the product coupling of the nucleus and electron angular momenta, i.e.,

$$
|\beta F m\rangle=\sum_{m_{I} m_{J}}\left\langle J m_{J} \operatorname{Im} m_{I} \mid F m\right\rangle\left|I m_{I}\right\rangle\left|\beta J m_{J}\right\rangle .
$$

Here, the quantities $\left\langle j_{1} m_{j_{1}} j_{2} m_{j_{2}} \mid j_{3} m_{j_{3}}\right\rangle$ stand for the ClebschGordan coefficients. After employing sum rules of ClebschGordan coefficients [47], we get

$$
\begin{aligned}
\left\langle\beta^{\prime} F^{\prime} m^{\prime} J^{\prime}\left|r_{\lambda}\right| \beta F m J\right\rangle \\
=(-1)^{F^{\prime}+I+F+1+J^{\prime}-m^{\prime}} \sqrt{\left[F, F^{\prime}\right]} \\
\quad \times\left(\begin{array}{ccc}
F^{\prime} & 1 & F \\
-m^{\prime} & \lambda & m
\end{array}\right)\left\{\begin{array}{ccc}
J & I & F \\
F^{\prime} & 1 & J^{\prime}
\end{array}\right\}\left\langle\beta^{\prime} J^{\prime}|| r \| \beta J\right\rangle,
\end{aligned}
$$

where the notation $\left[j_{1}, j_{2}, \ldots\right]$ is equal to $\left(2 j_{1}+1\right)\left(2 j_{2}+1\right) \ldots$ Since $r_{\lambda}$ does not act on the spin part of the wave function, the reduced matrix element $\left\langle J^{\prime}\left\|r_{\lambda}\right\| J_{i}\right\rangle$ is given by

$$
\begin{aligned}
\left\langle\beta^{\prime} J^{\prime}\|r\| \beta J\right\rangle= & (-1)^{J^{\prime}-1 / 2} \sqrt{\left[J, J^{\prime}\right]}\left(\begin{array}{ccc}
J^{\prime} & 1 & J \\
1 / 2 & 0 & -1 / 2
\end{array}\right) \\
& \times\left\langle n^{\prime}\|r\| n\right\rangle,
\end{aligned}
$$


provided that $L^{\prime}+L+1$ is even, where $L$ is the orbital angular momentum of the atomic state. Combining Eqs. (A4) and (A5) and rearranging the terms, the quantities $\mathcal{S}_{f v i}$ and $\Omega_{J_{i} J_{v} J_{f}}^{F_{i} F_{v} F_{f}}(\theta, \chi)$ of Eqs. (3) and (4) can be written as

$$
\begin{aligned}
\mathcal{S}_{f v i} & =\left\langle n_{f}\|r\| n_{\nu}\right\rangle\left\langle n_{\nu}\|r\| n_{i}\right\rangle=\int r^{3} R_{f} R_{\nu} d r \int r^{3} R_{\nu} R_{i} d r \\
& =-\frac{128 \sqrt{2}}{27\left(m_{\mu n} Z\right)^{2}}
\end{aligned}
$$

and

$$
\begin{aligned}
& \Omega_{J_{i} J_{v} J_{f}}^{F_{i} F_{v} F_{f}}\left(\theta, \chi, \eta, \boldsymbol{\varepsilon}_{2}\right) \\
&= {\left[J_{v}, F_{v}\right] \sqrt{\left[F_{f}, F_{i}, J_{f}, J_{i}\right]} } \\
& \times\left(\begin{array}{ccc}
J_{f} & 1 & J_{v} \\
1 / 2 & 0 & -1 / 2
\end{array}\right)\left(\begin{array}{ccc}
J_{v} & 1 & J_{i} \\
1 / 2 & 0 & -1 / 2
\end{array}\right) \\
& \times\left\{\begin{array}{ccc}
F_{f} & 1 & F_{v} \\
J_{v} & I & J_{f}
\end{array}\right\}\left\{\begin{array}{lll}
F_{v} & 1 & F_{i} \\
J_{i} & I & J_{v}
\end{array}\right\} \theta_{F_{f} F_{i}}^{F_{v}},
\end{aligned}
$$

with

$$
\begin{aligned}
\theta_{F_{f} F_{i}}^{F_{v}}= & \sum_{\lambda_{1}, \lambda_{2}} \sum_{m_{v}}(-1)^{\lambda_{1}+\lambda_{2}+m_{v}+m_{f}+1} \varepsilon_{1}^{\lambda_{1}} \varepsilon_{2}^{\lambda_{2} *} \\
& \times\left(\begin{array}{ccc}
F_{f} & 1 & F_{v} \\
-m_{f} & \lambda_{2} & m_{v}
\end{array}\right)\left(\begin{array}{ccc}
F_{v} & 1 & F_{i} \\
-m_{v} & \lambda_{1} & m_{i}
\end{array}\right) .
\end{aligned}
$$

The functions $R$ in Eq. (A6) stand for the radial nonrelativistic wave functions, which for the case of $2 s \rightarrow 2 p \rightarrow 1 s$ gives the numerical result shown on the right side of Eq. (A6). The quantity $m_{\mu n}$ is the ratio between the muon-nucleus reduced mass and the electron mass.

In case of incident linear polarized photons, the dipole radiation pattern of the scattered photon depends only on the
TABLE III. Values of the coefficients $a_{0}, a_{2}$, and $b_{2}$ corresponding to the parametrizations $\Lambda_{J_{i} J_{v}}^{F_{i} F_{v}}(\theta, \chi)=a_{0}+a_{2} P_{2}(\cos \gamma)$ and $\Xi_{J_{i} J_{v} J_{v^{\prime}}}^{F_{i} F_{v} F_{v^{\prime}}}(\theta, \chi)=b_{2} P_{2}(\cos \gamma)$.

\begin{tabular}{ccccccccc}
\hline \hline$I$ & $F_{i}$ & $F_{v}$ & $J_{v}$ & $F_{v^{\prime}}$ & $J_{v^{\prime}}$ & $a_{0}$ & $a_{2}$ & $b_{2}$ \\
\hline $1 / 2$ & 0 & 1 & $1 / 2$ & 1 & $3 / 2$ & $2 / 81$ & 0 & $-4 / 81$ \\
& 0 & 1 & $3 / 2$ & - & - & $4 / 81$ & $-2 / 81$ & - \\
& 1 & 1 & $1 / 2$ & 1 & $3 / 2$ & $4 / 81$ & 0 & $-2 / 81$ \\
& 1 & 1 & $1 / 2$ & 2 & $3 / 2$ & $4 / 81$ & 0 & $-2 / 27$ \\
1 & 1 & $3 / 2$ & 2 & $3 / 2$ & $2 / 81$ & $1 / 162$ & $-1 / 27$ \\
& 1 & 2 & $3 / 2$ & 1 & $3 / 2$ & $10 / 81$ & $-7 / 162$ & - \\
1 & $1 / 2$ & $1 / 2$ & $1 / 2$ & $3 / 2$ & $3 / 2$ & $4 / 729$ & 0 & $-8 / 729$ \\
& $1 / 2$ & $1 / 2$ & $3 / 2$ & $3 / 2$ & $1 / 2$ & $32 / 729$ & 0 & $-32 / 729$ \\
$1 / 2$ & $3 / 2$ & $3 / 2$ & - & - & $40 / 729$ & $-4 / 729$ & - \\
& $3 / 2$ & $1 / 2$ & $1 / 2$ & $3 / 2$ & $3 / 2$ & $32 / 729$ & 0 & $-32 / 3645$ \\
$3 / 2$ & $1 / 2$ & $1 / 2$ & $5 / 2$ & $3 / 2$ & $32 / 729$ & 0 & $-32 / 405$ \\
& $3 / 2$ & $1 / 2$ & $3 / 2$ & $3 / 2$ & $1 / 2$ & $4 / 729$ & 0 & $-4 / 729$ \\
$3 / 2$ & $1 / 2$ & $3 / 2$ & $3 / 2$ & $3 / 2$ & $4 / 729$ & 0 & $16 / 3645$ \\
$3 / 2$ & $1 / 2$ & $3 / 2$ & $5 / 2$ & $3 / 2$ & $4 / 729$ & 0 & $-4 / 405$ \\
$3 / 2$ & $3 / 2$ & $1 / 2$ & $3 / 2$ & $3 / 2$ & $40 / 729$ & 0 & $-128 / 3645$ \\
$3 / 2$ & $3 / 2$ & $1 / 2$ & $5 / 2$ & $3 / 2$ & $40 / 729$ & 0 & $-28 / 405$ \\
$3 / 2$ & $3 / 2$ & $3 / 2$ & $5 / 2$ & $3 / 2$ & $32 / 729$ & $64 / 18225$ & $-112 / 2025$ \\
$3 / 2$ & $5 / 2$ & $3 / 2$ & - & - & $4 / 27$ & $-28 / 675$ & - \\
\hline \hline
\end{tabular}

angle $\gamma$ between the incident polarization and the direction of the scattered photon, which is related to the previous angles by $\cos \gamma=\cos \chi \sin \theta \cdot \Lambda_{J_{i} J_{v}}^{F_{i} F_{v}}(\theta, \chi)$ and $\Xi_{J_{i} J_{v} J_{v^{\prime}}}^{F_{i} F_{v}}(\theta, \chi)$ are parametrized in terms of this angle $\gamma$ by $a_{0}+a_{2} P_{2}(\cos \gamma)$ and $b_{2} P_{2}(\cos \gamma)$, respectively. The respective coefficients calculated using Eq. (A7) are listed in Table III.
[1] F. Low, Phys. Rev. 88, 53 (1952).

[2] L. Labzowsky, V. Karasiev, and I. Goidenko, J. Phys. B 27, L439 (1994).

[3] L. N. Labzowsky, D. A. Solovyev, G. Plunien, and G. Soff, Phys. Rev. Lett. 87, 143003 (2001).

[4] L. Labzowsky, G. Schedrin, D. Solovyev, E. Chernovskaya, G. Plunien, and S. Karshenboim, Phys. Rev. A 79, 052506 (2009).

[5] R. C. Brown, S. Wu, J. V. Porto, C. J. Sansonetti, C. E. Simien, S. M. Brewer, J. N. Tan, and J. D. Gillaspy, Phys. Rev. A 87, 032504 (2013).

[6] A. Huber, B. Gross, M. Weitz, and T. W. Hänsch, Phys. Rev. A 59, 1844 (1999).

[7] M. Niering, R. Holzwarth, J. Reichert, P. Pokasov, T. Udem, M. Weitz, T. W. Hänsch, P. Lemonde, G. Santarelli, M. Abgrall, P. Laurent, C. Salomon, and A. Clairon, Phys. Rev. Lett. 84, 5496 (2000).

[8] C. G. Parthey, A. Matveev, J. Alnis, B. Bernhardt, A. Beyer, R. Holzwarth, A. Maistrou, R. Pohl, K. Predehl, T. Udem, T. Wilken, N. Kolachevsky, M. Abgrall, D. Rovera, C. Salomon, P. Laurent, and T. W. Hänsch, Phys. Rev. Lett. 107, 203001 (2011).
[9] U. D. Jentschura and P. J. Mohr, Can. J. Phys. 80, 633 (2002).

[10] L. N. Labzowsky, G. Schedrin, D. Solovyev, and G. Plunien, Can. J. Phys. 85, 585 (2007).

[11] S. G. Karshenboim and V. G. Ivanov, Astron. Lett. 34, 289 (2008).

[12] U. D. Jentschura, J. Evers, M. Haas, and C. H. Keitel, Phys. Rev. Lett. 91, 253601 (2003).

[13] P. A. Franken, Phys. Rev. 121, 508 (1961).

[14] R. Walkup, A. L. Migdall, and D. E. Pritchard, Phys. Rev. A 25, 3114 (1982).

[15] C. J. Sansonetti, C. E. Simien, J. D. Gillaspy, J. N. Tan, S. M. Brewer, R. C. Brown, S. Wu, and J. V. Porto, Phys. Rev. Lett. 107, 023001 (2011).

[16] M. Horbatsch and E. A. Hessels, Phys. Rev. A 82, 052519 (2010).

[17] A. Marsman, M. Horbatsch, and E. A. Hessels, Phys. Rev. A 86, 012510 (2012).

[18] A. Marsman, M. Horbatsch, and E. A. Hessels, Phys. Rev. A 86, 040501 (2012).

[19] A. Marsman, E. A. Hessels, and M. Horbatsch, Phys. Rev. A 89, 043403 (2014). 
[20] A. Marsman, M. Horbatsch, and E. A. Hessels, Phys. Rev. A 91, 062506 (2015).

[21] A. Marsman, M. Horbatsch, and E. A. Hessels, J. Phys. Chem. Ref. Data 44, 031207 (2015).

[22] D. C. Yost, A. Matveev, E. Peters, A. Beyer, T. W. Hänsch, and T. Udem, Phys. Rev. A 90, 012512 (2014).

[23] E. Borie, Ann. Phys. 327, 733 (2012).

[24] A. Antognini, F. Kottmann, F. Biraben, P. Indelicato, F. Nez, and R. Pohl, Ann. Phys. 331, 127 (2013).

[25] E. Milotti, At. Data Nucl. Data Tables 70, 137 (1998).

[26] L. Safari, P. Amaro, S. Fritzsche, J. P. Santos, S. Tashenov, and F. Fratini, Phys. Rev. A 86, 043405 (2012).

[27] L. Safari, P. Amaro, J. P. Santos, and F. Fratini, Radiat. Phys. Chem. 106, 271 (2015).

[28] A. P. Martynenko, Phys. Rev. A 71, 022506 (2005).

[29] A. Martynenko, JETP 106, 690 (2008).

[30] E. Borie, Phys. Rev. A 71, 032508 (2005).

[31] R. Pohl, A. Antognini, F. Nez, F. D. Amaro, F. Biraben, J. M. R. Cardoso, D. S. Covita, A. Dax, S. Dhawan, L. M. P. Fernandes, A. Giesen, T. Graf, T. W. Hänsch, P. Indelicato, L. Julien, C.-Y. Kao, P. Knowles, E.-O. Le Bigot, Y.-W. Liu, J. A. M. Lopes, L. Ludhova, C. M. B. Monteiro, F. Mulhauser, T. Nebel, P. Rabinowitz, J. M. F. dos Santos, L. A. Schaller, K. Schuhmann, C. Schwob, D. Taqqu, J. F. C. A. Veloso, and F. Kottmann, Nature (London) 466, 213 (2010).

[32] A. Antognini, F. Nez, K. Schuhmann, F. D. Amaro, F. Biraben, J. M. R. Cardoso, D. S. Covita, A. Dax, S. Dhawan, M. Diepold, L. M. P. Fernandes, A. Giesen, A. L. Gouvea, T. Graf, T. W. Hänsch, P. Indelicato, L. Julien, C.-Y. Kao, P. Knowles, F. Kottmann, E.-O. Le Bigot, Y.-W. Liu, J. A. M. Lopes, L. Ludhova, C. M. B. Monteiro, F. Mulhauser, T. Nebel, P. Rabinowitz, J. M. F. dos Santos, L. A. Schaller, C. Schwob, D. Taqqu, J. F. C. A. Veloso, J. Vogelsang, and R. Pohl, Science 339, 417 (2013).

[33] A. Antognini, F. Biraben, J. M. R. Cardoso, D. S. Covita, A. Dax, L. M. P. Fernandes, A. L. Gouvea, T. Graf, T. W. Hänsch, M. Hildebrandt, P. Indelicato, L. Julien, K. Kirch, F. Kottmann, Y. W. Liu, C. M. B. Monteiro, F. Mulhauser, T. Nebel, F. Nez, J. M. F. dos Santos, K. Schuhmann, D. Taqqu, J. F. C. A. Veloso, A. Voss, and R. Pohl, Can. J. Phys. 89, 47 (2011).

[34] T. Nebel, F. D. Amaro, A. Antognini, F. Biraben, J. M. R. Cardoso, D. S. Covita, A. Dax, L. M. P. Fernandes, A. L. Gouvea, T. Graf, T. W. Hänsch, M. Hildebrandt, P. Indelicato, L. Julien, K. Kirch, F. Kottmann, Y. W. Liu, C. M. B. Monteiro, F. Nez, J. M. F. d. Santos, K. Schuhmann, D. Taqqu, J. F. C. A. Veloso, A. Voss, and R. Pohl, Hyperfine Interact. 212, 195 (2012).

[35] R. Loudon, The Quantum Theory of Light (Oxford Science Publications, Oxford, 2000).
[36] A. I. Akhiezer and V. B. Berestetskii, Quantum Electrodynamics (Interscience Publishers, New York, 1965).

[37] L. Safari, P. Amaro, J. P. Santos, and F. Fratini, Phys. Rev. A 90, 014502 (2014).

[38] K. Pachucki, Phys. Rev. A 53, 2092 (1996).

[39] Elliptical polarization of the incident photon can only decrease the QI shift and will be reported elsewhere.

[40] Computer code MINUIT 5.34/18, Function Minimization and Error Analysis, CERN, Meyrin, Switzerland (2014).

[41] A. Antognini, F. D. Amaro, F. Biraben, J. M. R. Cardoso, C. A. N. Conde, D. S. Covita, A. Dax, S. Dhawan, L. M. P. Fernandes, and T. W. Hänsch, Opt. Commun. 253, 362 (2005).

[42] A. Antognini, K. Schuhmann, F. D. Amaro, F. Biraben, A. Dax, A. Giesen, T. Graf, T. W. Hänsch, P. Indelicato, L. Julien, K. Cheng-Yang, P. E. Knowles, F. Kottmann, E. Le Bigot, L. Yi-Wei, L. Ludhova, N. Moschuring, F. Mulhauser, T. Nebel, F. Nez, P. Rabinowitz, C. Schwob, D. Taqqu, and R. Pohl, IEEE J. Quantum Electron. 45, 993 (2009).

[43] J. Vogelsang, M. Diepold, A. Antognini, A. Dax, J. Götzfried, T. W. Hänsch, F. Kottmann, J. J. Krauth, Y.-W. Liu, T. Nebel, F. Nez, K. Schuhmann, D. Taqqu, and R. Pohl, Opt. Express 22, 13050 (2014).

[44] L. Ludhova, F. D. Amaro, A. Antognini, F. Biraben, J. M. R. Cardoso, C. A. N. Conde, D. S. Covita, A. Dax, S. Dhawan, L. M. P. Fernandes, T. W. Hänsch, V. W. Hughes, O. Huot, P. Indelicato, L. Julien, P. E. Knowles, F. Kottmann, J. A. M. Lopes, Y. W. Liu, C. M. B. Monteiro, F. Mulhauser, F. Nez, R. Pohl, P. Rabinowitz, J. M. F. dos Santos, L. A. Schaller, D. Taqqu, and J. F. C. A. Veloso, Nucl. Instrum. Meth. Phys. A 540, 169 (2005).

[45] L. M. P. Fernandes, F. D. Amaro, A. Antognini, J. M. R. Cardoso, C. A. N. Conde, O. Huot, P. E. Knowles, F. Kottmann, J. A. M. Lopes, L. Ludhova, C. M. B. Monteiro, F. Mulhauser, R. Pohl, J. M. F. d. Santos, L. A. Schaller, D. Taqqu, and J. F. C. A. Veloso, Journal of Instrumentation 2, P08005 (2007).

[46] M. Diepold, L. M. P. Fernandes, J. Machado, P. Amaro, M. Abdou-Ahmed, F. D. Amaro, A. Antognini, F. Biraben, T.-L. Chen, D. S. Covita, A. J. Dax, B. Franke, S. Galtier, A. L. Gouvea, J. Gtzfried, T. Graf, T. W. Hänsch, M. Hildebrandt, P. Indelicato, L. Julien, K. Kirch, A. Knecht, F. Kottmann, J. J. Krauth, Y.-W. Liu, C. M. B. Monteiro, F. Mulhauser, B. Naar, T. Nebel, F. Nez, J. P. Santos, J. M. F. dos Santos, K. Schuhmann, C. I. Szabo, D. Taqqu, J. F. C. A. Veloso, A. Voss, B. Weichelt, and R. Pohl, Rev. Sci. Instrum. 86, 053102 (2015).

[47] M. E. Rose, Elementary Theory of Angular Momentum (John Wiley, New York, 1957). 